\title{
Real and Complex Valued Ripplet-I Transform for Medical Image Denoising and Analysis of Thresholding Constants and Scales Effects
}

\author{
${ }^{1}$ Hüseyin Yaşar, ${ }^{2}$ Murat Ceylan \\ ${ }^{1}$ Ministry of Health of the Republic of Turkey, Ankara, Turkey; \\ ${ }^{2}$ Department of Electrical and Electronics Engineering, Selcuk University, Konya, Turkey; \\ mirhendise@gmail.com; mceylan@selcuk.edu.tr
}

\begin{abstract}
Medical image processing is an important diagnostic tool in the field of medical. Medical images might be affected by the noises that manipulate the resolution negatively during screening or transmission. These images need to be eliminated so as not to affect the diagnosis success negatively. In medical image denoising studies, using the multi-resolution analysis coefficients is a widely appreciated method. This study tested the success rate of real and complex valued ripplet-I transform for medical image denoising. Thanks to this study, the complex version of the newly suggested ripplet-I transform whose real version was used formerly in various studies was used in a medical image denoising application the first time. In the study tested with 40 liver images, 40 retinal images and 322 mammographic images, peak signal-to-noise ratio (PSNR), mean structural similarity index (MSSIM) and feature similarity index (FSIM) were utilized to compare the successes of image denoising. In the wake of study, it was seen that the complex valued ripplet-I (CVR-I) transform gave better results than the real valued ripplet-I (RVR-I) transform when used in the same image denoising algorithm. This study also examined the effects that the changes in scale and thresholding constant values have on the medical image denoising results, thus making this study appear as a guideline.
\end{abstract}

Keywords: Real and complex valued ripplet-I transform; Medical image denoising; Thresholding constant; Peak signal-to-noise ratio (PSNR); Mean structural similarity index (MSSIM); Feature similarity index (FSIM).

\section{Introduction}

Image denoising is a field of image processing upon which many researches were conducted. Important developments also took place in this field as parallel with the development of multi-resolution analyses. Firstly, the wavelet transform was put forth, creating the basis of multi-resolution analyses and various successful denoising applications followed through by using the wavelet transform and different algorithms [1-9].

The wavelet transform only analyzing in certain aspects has caused a big flaw when representing images. To eradicate this flaw, Candes and Donoho put forth the ridgelet transform that can analyze in 
Hüseyin Yaşar, Murat Ceylan; Real and Complex Valued Ripplet-I Transform for Medical Image Denoising and Analysis of Thresholding Constants and Scales Effects . Journal of Biomedical Engineering and Medical Imaging, Volume 4, No 2, April (2017), pp 10-30

various aspects [10]. The curvelet transform based on the ridgelet transform and, which has created at least as big an impact as the wavelet transform, was defined again by Candes and Donoho [11]. Starck et al. suggested that the curvelet transform which later would be known as the first-generation curvelet transform gave better results for denoising than the wavelet transform [12]. Following this, it was seen that the curvelet transform created better results than the wavelet transform in image denoising applications in many studies [13-19]. Along with the curvelet transform having created such big impact, its first iteration has created too much and non-used data. This increased the process load and decreased the process speed. To eliminate this negative outcome, studies towards the definition of second generation curvelet transform was conducted by Candes et al. [20, 21].

The biggest disadvantage of the first iterations of multi-resolution analyses is the lack of phase information. Lack of phase information inhibits the number of analyzed aspects. Lawton and Lina showed the applicability of the wavelet transform in the complex form [22, 23]. Thanks to that Selesnick et al. defined the dual-tree discrete complex wavelet transform with two similar models (Kingsbury's and Selesnick's) a study was put forth towards overcoming the issue of inhibited orientation of the real valued wavelet transform [24]. Complex valued wavelet transform gave better results in the denoising applications compared to real valued wavelet transform [25-27].

In 2005, Candes et al. defined the fast discrete curvelet transform based on the second generation curvelet transform [28]. This study utilized complex curvelet coefficients. In studies produced by Neelamani et al., the complex form of curvelet transform was used [29-31]. In 2010, Yan et al. made image restoration using the complex form of the transform [32].

In 2010, Xu et al. described the ripplet-I transform which is a higher dimensional generalization of the curvelet transform (by adding support and degree parameters), designed to represent images or twodimensional signals at different scales and different directions [33]. Per this study, the denoising application was conducted and the denoising results for some various values of the support, degree parameters were compared. After the real valued ripplet-I (RVR-I) transform was put forth, it began to be used widely in the literature. Also, in 2014, by using RVR-I coefficients, Gupta et al. realized the image denoising [34]. In 2016, Yaşar and Ceylan described the comlex ripplet-I transform [35]. In same year, they realized automatic blood vessel extraction in retinal images using complex ripplet-I transform [36].

During screening or transmission, Medical images might be affected by images that have a negative impact on the resolution. These images need to be eliminated so as not to affect the diagnosis success negatively. In medical images, denoising aims to obtain the image or protect the originality of the image by eradicating the noises that occur during transmission.

This study helped us realize the medical image denoising application for different scale, support, degree, thresholding constant values by using RVR-I and complex valued ripplet-I (CVR-I) transform. The effects that the changes in scale, support, degree, thresholding constant values have on denoising were fully analyzed, thus making this study appear as a guideline. Thanks to this study, the complex version of the newly suggested ripplet-I transform whose real version was used formerly in various studies was used in a medical image denoising application the first time. The numerical results in this study were obtained from MSc thesis study of Yaşar [37]. 


\section{Methods}

\subsection{Ripplet-I Transform}

Ripplet-I transform generalizes curvelet transform by adding two parameters, support $(c)$ and degree (d). These new parameters, $c$ and $d$, provide ripplet-I with anisotropic capability of representing 2D singularities along arbitrarily shaped curves [33]. The ripplet function is defined in Equation (1).

$\rho_{\vec{a} \overrightarrow{0}(x)}$ is the ripplet element function, $R_{\theta}=\left(\begin{array}{cc}\cos \theta & \sin \theta \\ -\sin \theta & \cos \theta\end{array}\right)$ is the rotation matrix, $\vec{x}$ and $\vec{b}$ are 2D vectors in Equation (1).

$$
\rho_{a \vec{b} \theta}(\vec{x})=\rho_{a \overrightarrow{0} 0}\left(R_{\theta}(\vec{x}-\vec{b})\right)
$$

The element ripplet function $\rho_{\overrightarrow{a 00}}(x)$ with scale parameter $a$ is defined in the frequency domain in polar coordinates [33].

$$
\hat{\rho_{\theta}}(r, \omega)=\frac{1}{\sqrt{c}} a^{\frac{1+d}{2 d}} W(a \cdot r) V\left(\frac{a^{\frac{1}{d}}}{c \cdot a} \cdot \omega\right)
$$

The discrete transform takes as input data defined on a Cartesian grid and outputs a collection of coefficients. For the scale parameter $a$, we sample at dyadic intervals. The position parameter $\vec{b}$ and rotation parameter $\theta$ are sampled at equal-spaced intervals. $a, \vec{b}$ and $\theta$ are substituted with discrete parameters $a_{j}, \vec{b}_{k}$ and $\theta_{l}$, which satisfy that $a_{j}=2^{-j}, \vec{b}_{k}=\left[c \cdot 2^{-j} \cdot k_{1}, 2^{-j / d} \cdot k_{2}\right]^{T}$ and $\theta_{l}=\frac{2 \pi}{c} \cdot 2^{-|j(1-1 / d)|} \cdot l$ where $\vec{k}=\left[k_{1}, k_{2}\right]^{T},(\cdot)^{T}$ denotes the transpose of a vector and $j, k_{1}, k_{2}, l \in Z$. The frequency response of ripplet function is given as $(d=n / m ; n, m \neq 0 \in Z)$ [33].

$$
\hat{\rho}_{j}(r, \omega)=\frac{1}{\sqrt{c}} a^{\frac{m+n}{2 n}} W\left(2^{-j} \cdot r\right) V\left(\frac{2^{-j\left|\frac{m-n}{n}\right|}}{c} \cdot \omega-l\right)
$$

where $W$ and $V$ satisfy the following conditions

$$
\sum_{j=0}^{\infty}\left|W\left(2^{-j} \cdot r\right)\right|^{2}=1 \sum_{l=-\infty}^{+\infty}\left|V\left(\frac{2^{-j|1-1 / d|}}{c} \cdot \omega-l\right)\right|^{2}=1
$$

Equations given between Equality (1) and (4) define the curvelet transform for values $c=1, d=2$.

The goal here is to find a digital implementation of the discrete ripplet-I transform, whose coefficients are now given by

$$
r_{j, k, l}:=\left\langle f \cdot \rho_{j, k, l}^{D}\right\rangle=\int_{R^{2}} \hat{f}(v) \tilde{\rho}_{j}^{D}\left(S_{\theta_{j}}^{-1} v\right) e^{i S_{\theta_{j}}^{-T} m \cdot v} d v
$$


Hüseyin Yaşar, Murat Ceylan; Real and Complex Valued Ripplet-I Transform for Medical Image Denoising and Analysis of Thresholding Constants and Scales Effects. Journal of Biomedical Engineering and Medical Imaging, Volume 4, No 2, April (2017), pp 10-30

where $S_{\theta}:=\left(\begin{array}{cc}1 & 0 \\ -\tan \theta & 1\end{array}\right)$ is the shear matrix and $m \approx\left[c \cdot 2^{-j} \cdot k_{1}, 2^{-j / d} \cdot k_{2}\right]$.

The operations after this stage are conducted as defined earlier by fast discrete curvelet transform [28]. The ripplet-I via wrapping are described below with the basic steps. $\tilde{U}_{j, l}\left[n_{1}, n_{2}\right]$ reindexing the samples array by wrapping around $\mathrm{a} \approx c \cdot 2^{j} \times 2^{j / d}$ (width $\left.\approx \mathrm{c} \cdot(\text { length })^{d}\right)$ rectangle centered at the origin [36].

Step 1: Compute 2D FFT coefficients to obtain Fourier samples $\hat{f}\left[n_{1}, n_{2}\right]$.

Step 2: Interpolation, for each scale and angle pair (j,l), $\tilde{U}_{j, l}\left[n_{1}, n_{2}\right] \hat{f}\left[n_{1}, n_{2}\right]$.

Step 3: Wrap result of step 2 around the origin and obtain $\tilde{f}\left[n_{1}, n_{2}\right]=W\left(\tilde{U}_{j, l} \tilde{f}\right)\left[n_{1}, n_{2}\right]$ where the range $n_{1}$ and $n_{2}$ are $0 \leq n_{1} \leq L_{1, j}$ and $0 \leq n_{2} \leq L_{2, j}$ respectively.

Step 4: Implement the inverse 2D FFT for each $\tilde{f}_{j, l}$ to obtain the discrete coefficients.

\subsection{Complex Valued Ripplet-I Transform}

Continuous ripplet-I as just constructed are complex valued. It is easy to obtain real-valued ripplet-I by working on the symmetrized version $\hat{\rho}_{j}(r, \omega)+\hat{\rho}_{j}(r, \omega+\pi)$. Equation (6) refers to the imaginary part of the CVR-I transform [35, 36].

$$
\hat{\rho}_{\theta}(r, \omega+\pi)=\frac{1}{\sqrt{c}} a^{\frac{1+d}{2 d}} W(a \cdot r) V\left(\frac{a^{\frac{1}{d}}}{c \cdot a} \cdot(\omega+\pi)\right)
$$

The discrete ripplet-I transform coefficients are complex-valued, but a real-valued discrete ripplet-I transform with the same redundancy factor can be easily obtained by properly combining coefficients at orientations $\theta_{l}$ and $\theta_{l}+\pi$.

Coefficients of CVR-I can be expressed as follows. $C V R-I_{R}$ and $C V R-I_{I}$ are real and the imaginary coefficients, respectively.

$$
C V R-I=C V R-I_{R}+j C V R-I_{I}
$$

Figure 1 shows the formation of complex valued ripplet-I coefficients in the real and imaginary coefficients.

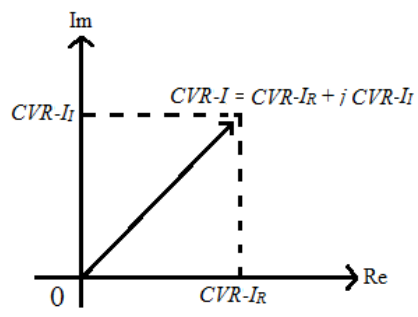

Figure 1. Complex valued ripplet-I coefficients. 
In Figure 2, images are given showing a real and imaginary ripplet-I coefficient for $c=1$ and $d=3$ values of Lena's [38] image and the CVR-I coefficient images obtained by combining these coefficients using Equation (7) [35].
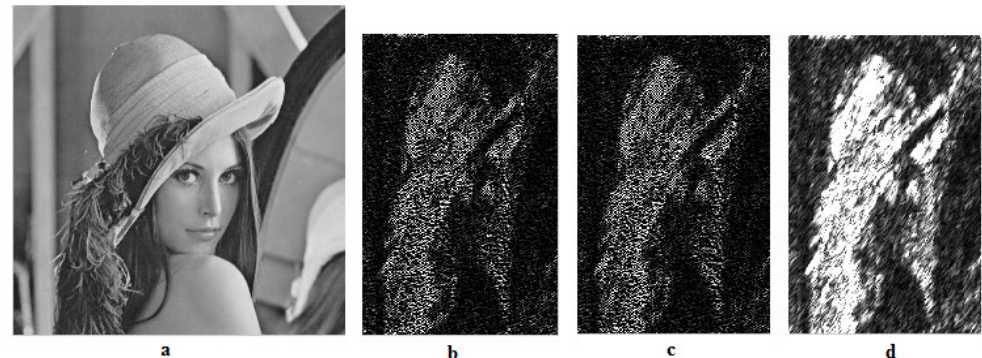

Figure 2. a) Original Lena image b) Real ripplet-I coefficient $(c=1, d=3) c$ ) Imaginary ripplet-I coefficient ( $c=1$, $d=3)$ d) CVR-I coefficient $(c=1, d=3)$.

In this study, the MATLAB codes located in curvelab [39] (fast discrete curvelet transform via wedge wrapping) were used by adapting to ripplet-I transform. This study helped us produce RVR-I and CVR-I coefficients for different scale, support, degree values. Support and degree parameters defined as the generalization of the ripplet-I transform and the curvelet transform make the decompositions used in the curvelet transform change. Decompositions used for different scale, support, degree values in the study are given in Table 1.

Table 1. Decompositions for different (a) scale, $(c)$ support and $(d)$ degree.

\begin{tabular}{|c|c|c|c|c|}
\cline { 2 - 5 } \multicolumn{1}{c|}{} & $c=1, d=2$ & $c=1, d=3$ & $c=1, d=4$ & $c=1, d=5$ \\
\hline$a=4$ & {$[1,2,2]$} & {$[1,2,2]$} & {$[1,2,4]$} & {$[1,2,4]$} \\
\hline$a=5$ & {$[1,2,2,4]$} & {$[1,2,2,4]$} & {$[1,2,4,8]$} & {$[1,2,4,8]$} \\
\hline$a=6$ & {$[1,2,2,4,4]$} & {$[1,2,4,4,8]$} & {$[1,2,4,8,8]$} & {$[1,2,4,8,16]$} \\
\hline$a=7$ & {$[1,2,2,4,4,8]$} & {$[1,2,4,4,8,16]$} & {$[1,2,4,8,8,16]$} & {$[1,2,4,8,16,16]$} \\
\hline
\end{tabular}

\subsection{Denoising Algorithm}

Generally, a noisy image $I_{n}$ can be denoted as below. Where $I$ is the original image, $N$ is noise and $N_{r}$ random noise :

$$
I_{n}=I+N=I+\operatorname{sigma}^{*} N_{r}
$$

Here, sigma is the noise ratio which is an important factor to specify the amount of noise that will be added to the image. If sigma value increases, the image will be much more noisy. In our study, we have used three sigma values $(15,25,35)$ for random noise.

Curvelet shrinkage image denoising method is an algorithm for denoising using curvelet coefficients. This algorithm can realized using ripplet-I coefficients. In the wavelet domain, there are two common shrinkage methods, namely, the hard-thresholding and the soft-thresholding approach. In this study, hard-thresholding (Equation 9) is used.

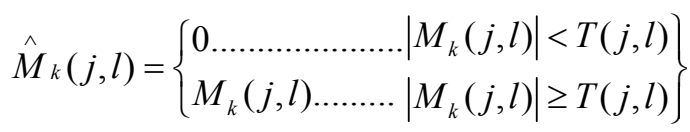


Hüseyin Yaşar, Murat Ceylan; Real and Complex Valued Ripplet-I Transform for Medical Image Denoising and Analysis of Thresholding Constants and Scales Effects . Journal of Biomedical Engineering and Medical Imaging, Volume 4, No 2, April (2017), pp 10-30

Where $T(j, l)$ is the threshold function and $M_{k}(j, l)$ is a ripplet-I coefficient dependent on scale index $j$ and angle index $l$, and $\hat{M}_{k}(j, l)$ denotes the ripplet-I coefficients processed. The threshold function $T(j, l)$ is defined as [40].

$$
T(j, l)=k \cdot \varepsilon_{(j, l)} \cdot \sigma
$$

$\varepsilon_{(j, l)}$ denotes the average energy distribution of white noise in ripplet-I coefficient on scale $j$ and angle $l$; $\sigma$ is defined as the standard deviation of white noise. $k$ is called as thresholding constant. The thresholding constant value is used generally as " 3 " in studies. Along with this, it is also known that per different values of the thresholding constant, the denoising results get affected. Instead of using a stable thresholding constant in this study, a number of thresholding constant values were used for the image denoising results; increasing or decreasing by " 0.1 " between a range of " 2.1 " and " 3.4 ".

Denoising processes for the CVR-I transform are realized with the same method. However, the image matrix elements obtained by reversing the transform also by using denoised coefficients consist of complex numbers. Absolute values of the matrix elements belonging to the re-obtained image after denoising were calculated by using Equation (11) and these results were used in PSNR calculations.

$$
\text { image }_{(a, b)}=\sqrt{\left(\operatorname{image}_{R_{(a, b)}}\right)^{2}+\left(\operatorname{image}_{I_{(a, b)}}\right)^{2}}
$$

\subsection{Used Data}

Medical images can be obtained through various methods such as roentgen, computed tomography, magnetic resonance imaging. Target body part, where the medical imaging is applied to, and the image properties belonging to this part affect the noise formation and the success rate of denoising. Thus, in order to increase the reliability of the results of medical image denoising, many images different from one another are used. Three different types of medical images as liver image, retinal image and mammographic image were utilized.

\subsubsection{Liver Images}

Liver MR images are an important diagnostic tool in detecting liver diseases such as cyst, hamartoma, hemangioma, focal nodular hyperplasia and adenoma. In distinguishing these diseases from each other, the contrast differences that occur in images in different phases of liver MR imaging are used. Denoising is of paramount importance as it negatively affects the diagnosis rate due to it occurring on the image and changing the contrast.

In this study, 40 liver MR images were used for medical image denoising. Images used in the study were gathered from Selcuk University Faculty of Medicine (Department of Radiology). These JPG-format images are in size $512 \times 512$.

\subsubsection{Retinal images}

Retinal fundus images are used frequently in detecting impairments associated with systematic diseases such as diabetic retinopathy, macula diseases and retinal tears. In detecting such diseases, segmentation of blood vessel structures or ruptured areas on the general retinal image is required. Noises that will occur on retinal images make distinguishing these structures from each other more difficult. 
In this study, 40 retinal fundus images belonging to the DRIVE [41] database were used for medical image denoising. These images are in $584 \times 565$ size and JPEG format. DRIVE database images are arranged as size of the $512 \times 512$. Processes benefitted from the green color space of the images belonging to the DRIVE database that has three different color dimensions as red, green and blue.

\subsubsection{Mammographic images}

Mammographic images are used when detecting mammographic abnormalities such as calcification, architectural distortion and asymmetry. Mammographic images are different from liver and retinal images in terms of the structure. Pixel values belonging to the breast tissue are very close to each other in mammographic images. This makes the segmentation of abnormal areas more difficult. Also the pixel values belonging to the breast tissue being very close to each other makes distinguishing noises and the breast tissue from one another in the image harder as well.

322 PGM-format mammographic images used in this study for image denoising belong to MIAS [42] database. The images areas are defined in size of $512 \times 512$ from the images whose original sizes are $1024 \times 1024$.

\subsection{Evaluation Criteria}

PSNR is a common method for comparison of image quality. However, usage of single comparison criteria is not a true approach for medical image comparison. For testing the reliability of obtained results in this study, two different comparison criteria (MSSIM [43, 44] and FSIM [45]) are used.

\subsubsection{Peak signal-to-noise ratio (PSNR)}

Mean squared error (MSE) calculates the square of the numeric difference between two images. Given a reference image $f$ and a test image $g$, both $\mathrm{M} \times \mathrm{N}$ sized, the MSE between $f$ and $g$ is defined by:

$$
\operatorname{MSE}(f, g)=\frac{1}{M N} \cdot \sum_{i=1}^{M} \sum_{j=1}^{N}\left(f_{i, j}-g_{i, j}\right)^{2}
$$

PSNR is an objective performance criterion which ensures to score the loss of information operations caused in dB. Given a reference image $f$ and a test image $g$ ( 8 bit), both $\mathrm{M} \times \mathrm{N}$ sized, the MSE between $f$ and $g$ is defined by:

$$
\operatorname{PSNR}(f, g)=10 \cdot \log _{10}\left(255^{2} / \operatorname{MSE}(f, g)\right)
$$

The PSNR value approaches infinity as the MSE approaches zero; this shows that a higher PSNR value provides a higher image quality. At the other tip of the scale, a small value of the PSNR implies high numerical distinctions between images.

\subsubsection{Mean structural similarity index (MSSIM)}

Structural similarity index (SSIM) of given two images $x$ and $y$, combines three components. They are called luminance distortion term $l(x, y)$, contrast distortion term $c(x, y)$ and correlation term $s(x, y)$. While $c_{1}, c_{2}$ and $c_{3}$ are constants, these components are denoted as [43]:

$$
l(x, y)=\frac{2 \overline{x y}+c_{1}}{(\bar{x})^{2}+(\bar{y})^{2}+c_{1}}
$$




$$
\begin{aligned}
& c(x, y)=\frac{2 s_{x} s_{y}+c_{2}}{s_{x}{ }^{2}+s_{y}{ }^{2}+c_{2}} \\
& s(x, y)=\frac{s_{x, y}+c_{3}}{2 s_{x} s_{y}+c_{2}}
\end{aligned}
$$

where $\bar{x}, \bar{y}, s_{x}^{2}, s_{y}^{2}$ and $s_{x, y}$ are the local mean of $x$ and $y$, the local variance of $x$ and $y$, and the covariance between $x$ and $y$, respectively.

Given $S_{1}(x, y)$ and $S_{2}(x, y)$ are the similarity maps computed by a local window. If $c_{3}=c_{2} / 2, \mathrm{SSIM}$ of given two images are calculated as:

$$
\begin{aligned}
& \operatorname{SSIM}=S_{1}(x, y) \cdot S_{2}(x, y) \\
& S_{1}(x, y)=l(x, y) \\
& S_{2}(x, y)=c(x, y) \cdot s(x, y)
\end{aligned}
$$

SSIM is processed on local regions using a sliding window [44]. This procedure is implemented from topleft to bottom-right corner of the images till all image is operated. While $M$ is the number of windows, MSSIM is denoted as:

$$
\operatorname{MSSIM}(x, y)=\frac{1}{M} \sum_{j=1}^{M} \operatorname{SSIM}\left(x_{j}, y_{j}\right)
$$

The more similar the images are to each other, the closer MSSIM is to 1.

\subsubsection{Feature Similarity Index (FSIM)}

Another novel performance evaluation method is FSIM which is calculated by using a value called phase congruence (PC) and a specified similarity measure (S) based on gradient magnitude (GM) and PC. Instead of determining sharp changes in intensity of images directly, PC model identifies the features where the Fourier components are maximum in phase [45].

GM is calculated by using Gradient operators like Sobel, Prewitt and Scharr operators. GM of an image $f(x, y)$ is stated as:

$$
G=\sqrt{G_{x}^{2}+G_{y}^{2}}
$$

where $G_{x}$ and $G_{y}$ are the partial derivatives along horizontal and vertical directions of the image. $S_{L}(x)$ is the similarity index which is based on PC and GM, between two images in Equation (20).

$$
S_{L}(x)=\left[S_{P C}(x)\right] \cdot\left[S_{G}(x)\right]
$$

FSIM is defined as Equation (21) while $\Omega$ states the whole image and is the $P C_{m}(x)$ is the PC.

$$
F S I M=\frac{\sum_{x \in \Omega} S_{L}(x) P C_{m}(x)}{\sum_{x \in \Omega} P C_{m}(x)}
$$

\section{Experiments and Results}

Medical image denoising application was realized by using RVR-I and CVR-I coefficients in this study. This study, primarily, went through the application of random noise possessing three different sigma values 
$(15,25,35)$ on the original images so as to obtain the noised images to be utilized. Noises on the obtained RVR-I and CVR-I coefficients were cleaned up with the hard-thresholding shrinkage image denoising method. The cleaning process was repeated starting from the thresholding constant 2.1 and ending with 3.4 as it increased 0.1 at each go. By using coefficients whose obtained noise had been cleaned up, ripplet-I transform was reversed and the images were re-formed. Matrix elements of the new image to be obtained by using CVR-I transform consist from complex numbers. Absolute values of the image matrix elements were calculated using Equation (11) and the processes utilized these values. PSNR, MSSIM and FSIM values between the original image and denoised images were calculated in the study as final. To increase the consistency of the results, these processes were repeated for each image 25 times and their averages were then calculated.

\subsection{Liver Image Denoising}

In the wake of study, the best denoising PSNR results obtained with 40 liver MR images for three different sigma values $(15,25,25)$ were given in Table $2-4$. Upon examining Table 2-4, the most successful denoising results were obtained so long as the scale value was " 5 ". Also it was seen that the thresholding constant, which is an important factor in denoising applications, requires to be used differently in processes conducted with RVR-I and CVR-I coefficients. The most successful results were obtained for the values between "2.8" and "3.4" of thresholding constant in the denoising application conducted with RVR-I coefficients for these images. In the denoising application conducted with CVR-I coefficients, it was observed that the results obtained between these values were lower compared to the application results conducted with RVR-I coefficients. In the denoising application conducted with CVR-I coefficients, the most successful results were obtained for the values between "2.2" and "2.6" of thresholding constant. Also, the PSNR values obtained within this range were the best results achieved in these denoising applications.

A number of original liver MR images used in the processes, their noise-applied states for three different sigma values and their re-obtained states after denoising application for different support, degree values are shown in Figure 3.

Table 2. Results Of PSNR For Liver Images (Sigma=35, A (Scale), C (Support), D (Degree) And K (Thresholding Constant)).

\begin{tabular}{|c|c|c|c|c|c|c|c|c|}
\cline { 2 - 8 } \multicolumn{1}{c|}{} & \multicolumn{4}{c|}{ Reel valued ripplet-I transform } & \multicolumn{4}{c|}{ Complex valued ripplet-I transform } \\
\cline { 2 - 9 } \multicolumn{1}{c|}{} & $c=1, d=2$ & $c=1, d=3$ & $c=1, d=4$ & $c=1, d=5$ & $c=1, d=2$ & $c=1, d=3$ & $c=1, d=4$ & $c=1, d=5$ \\
\hline$a=7$ & $34.05(k=3.1)$ & $33.74(k=3.1)$ & $33.57(k=3.1)$ & $33.58(k=3.1)$ & $34.36(k=2.4)$ & $34.00(k=2.4)$ & $33.81(k=2.4)$ & $33.79(k=2.4)$ \\
\hline$a=6$ & $34.32(k=3.1)$ & $34.17(k=3.1)$ & $34.13(k=3.1)$ & $34.12(k=3.1)$ & $34.63(k=2.4)$ & $34.45(k=2.4)$ & $34.42(k=2.5)$ & $34.41(k=2.5)$ \\
\hline$a=5$ & $34.46(k=3.2)$ & $34.43(k=3.2)$ & $34.44(k=3.2)$ & $34.44(k=3.2)$ & $34.75(k=2.5)$ & $34.71(k=2.5)$ & $34.71(k=2.5)$ & $34.71(k=2.5)$ \\
\hline$a=4$ & $33.27(k=3.4)$ & $33.27(k=3.4)$ & $33.27(k=3.4)$ & $33.27(k=3.4)$ & $33.44(k=2.6)$ & $33.44(k=2.6)$ & $33.44(k=2.6)$ & $33.44(k=2.6)$ \\
\hline
\end{tabular}


Hüseyin Yaşar, Murat Ceylan; Real and Complex Valued Ripplet-I Transform for Medical Image Denoising and Analysis of Thresholding Constants and Scales Effects . Journal of Biomedical Engineering and Medical Imaging, Volume 4, No 2, April (2017), pp 10-30

Table 3. Results of PSNR for liver images (Sigma=25, $a$ (Scale), $c$ (Support), $d$ (Degree) and $k$ (Thresholding Constant)).

\begin{tabular}{|c|c|c|c|c|c|c|c|c|}
\cline { 2 - 8 } \multicolumn{1}{c|}{} & \multicolumn{4}{c|}{ Reel valued ripplet-I transform } & \multicolumn{4}{c|}{ Complex valued ripplet-I transform } \\
\cline { 2 - 9 } \multicolumn{1}{c|}{} & $c=1, d=2$ & $c=1, d=3$ & $c=1, d=4$ & $c=1, d=5$ & $c=1, d=2$ & $c=1, d=3$ & $c=1, d=4$ & $c=1, d=5$ \\
\hline$a=7$ & $35.67(k=3.0)$ & $35.38(k=3.0)$ & $35.11(k=3.0)$ & $35.07(k=3.0)$ & $35.98(k=2.4)$ & $35.62(k=2.3)$ & $35.37(k=2.3)$ & $35.30(k=2.3)$ \\
\hline$a=6$ & $35.91(k=3.0)$ & $35.74(k=3.0)$ & $35.67(k=3.0)$ & $35.66(k=3.0)$ & $36.24(k=2.4)$ & $36.03(k=2.4)$ & $35.96(k=2.4)$ & $35.95(k=2.4)$ \\
\hline$a=5$ & $36.08(k=3.1)$ & $36.05(k=3.1)$ & $36.05(k=3.1)$ & $36.05(k=3.1)$ & $36.36(k=2.4)$ & $36.34(k=2.4)$ & $36.34(k=2.4)$ & $36.34(k=2.4)$ \\
\hline$a=4$ & $35.36(k=3.3)$ & $35.35(k=3.3)$ & $35.35(k=3.3)$ & $35.35(k=3.3)$ & $35.56(k=2.5)$ & $35.56(k=2.5)$ & $35.56(k=2.5)$ & $35.56(k=2.5)$ \\
\hline
\end{tabular}

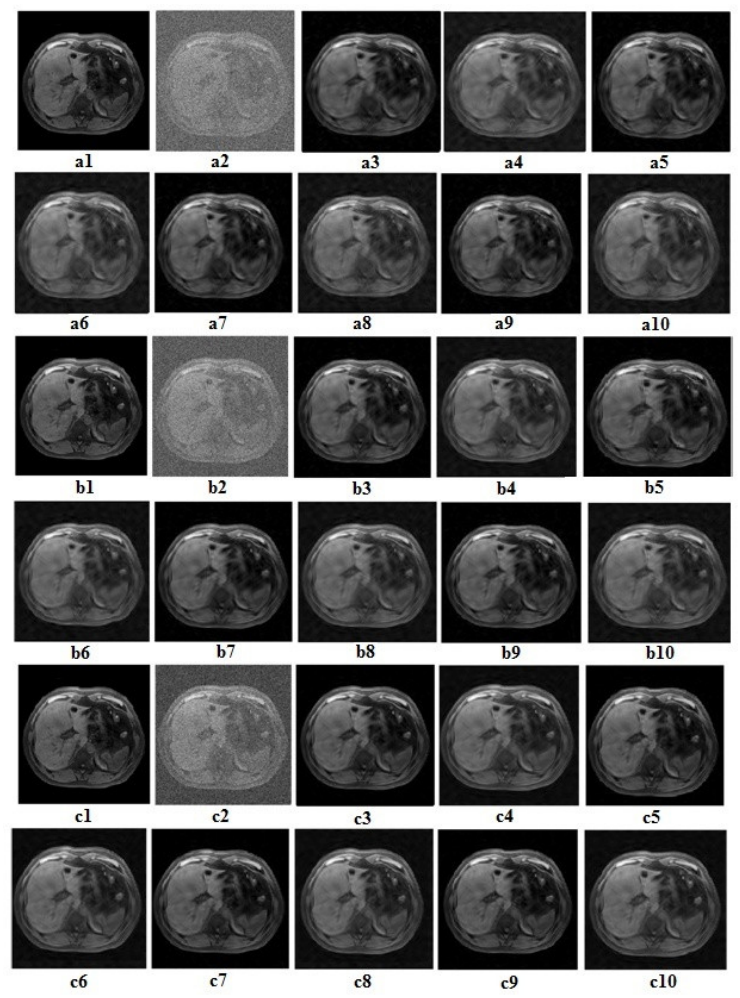

Figure 3. a1) Original liver image a2) Noisy image (Sigma=35) a3) Denoising with RVR-I (c=1, $d=2)$ a4) Denoising with CVR-I (c=1, $d=2)$ a5) Denoising with RVR-I $(c=1, d=3)$ a6) Denoising with CVR-I $(c=1, d=3)$ a7) Denoising with RVR-I (c=1, $d=4)$ a8) Denoising with CVR-I (c=1, $d=4)$ a9) Denoising with RVR-I $(c=1, d=5)$ a10) Denoising with CVR-I (c=1, $d=5)$ b1) Original liver image b2) Noisy image (Sigma=25) b3) Denoising with RVR-I (c=1, $d=2)$ b4) Denoising with CVR-I (c=1, $d=2)$ b5) Denoising with RVR-I (c=1, $d=3)$ b6) Denoising with CVR-I $(c=1, d=3)$ b7) Denoising with RVR-I (c=1, $d=4)$ b8) Denoising with CVR-I (c=1,d=4) b9) Denoising with RVR-I $(c=1, d=5)$ b10) Denoising with CVR-I (c=1, $d=5)(1)$ Original liver image c2) Noisy image (Sigma=15) c3) Denoising with RVR-I $(c=1, d=2)$ c4) Denoising with CVR-I $(c=1, d=2)$ c5) Denoising with RVR-I (c=1, $d=3)$ c6) Denoising with CVR-I $(c=1, d=3)$ c7) Denoising with RVR-I $(c=1, d=4) c 8)$ Denoising with CVR-I $(c=1, d=4)$ c9) Denoising with RVR-I $(c=1, d=5)$ c10) Denoising with CVR-I $(c=1, d=5)$. 
Table 4. Results of PSNR for liver Images (Sigma=15, $a$ (Scale), $c$ (Support), $d$ (Degree) and $k$ (Thresholding Constant)).

\begin{tabular}{|c|c|c|c|c|c|c|c|c|}
\cline { 2 - 8 } \multicolumn{1}{c|}{} & \multicolumn{4}{|c|}{ Reel valued ripplet-I transform } & \multicolumn{4}{c|}{ Complex valued ripplet-I transform } \\
\cline { 2 - 9 } \multicolumn{1}{c|}{} & $c=1, d=2$ & $c=1, d=3$ & $c=1, d=4$ & $c=1, d=5$ & $c=1, d=2$ & $c=1, d=3$ & $c=1, d=4$ & $c=1, d=5$ \\
\hline$a=7$ & $38.23(k=2.9)$ & $37.92(k=2.9)$ & $37.62(k=2.8)$ & $37.52(k=2.8)$ & $38.49(k=2.3)$ & $38.18(k=2.3)$ & $37.87(k=2.2)$ & $37.73(k=2.2)$ \\
\hline$a=6$ & $38.51(k=3.0)$ & $38.27(k=2.9)$ & $38.13(k=2.9)$ & $38.13(k=2.9)$ & $38.79(k=2.3)$ & $38.52(k=2.3)$ & $38.38(k=2.3)$ & $38.38(k=2.3)$ \\
\hline$a=5$ & $38.66(k=3.0)$ & $38.60(k=3.0)$ & $38.61(k=3.0)$ & $38.61(k=3.0)$ & $38.93(k=2.3)$ & $38.86(k=2.3)$ & $38.86(k=2.3)$ & $38.86(k=2.3)$ \\
\hline$a=4$ & $38.35(k=3.0)$ & $38.35(k=3.0)$ & $38.35(k=3.0)$ & $38.35(k=3.0)$ & $38.54(k=2.4)$ & $38.54(k=2.4)$ & $38.54(k=2.4)$ & $38.54(k=2.4)$ \\
\hline
\end{tabular}

\subsection{Retinal Image Denoising}

In the wake of study, the best denoising PSNR results obtained with 40 retinal images for three different sigma values $(15,25,25)$ were given in Table 5-7. Upon examining Table 5-7, the most successful denoising results were obtained so long as the scale value was " 5 ". Also it was seen that the thresholding constant, which is an important factor in denoising applications, requires to be used differently in processes conducted with RVR-I and CVR-I coefficients. The most successful results were obtained for the values between "2.7" and "3.3" of thresholding constant in the denoising application conducted with RVR-I coefficients for these images. In the denoising application conducted with CVR-I coefficients, it was observed that the results obtained between these values were lower compared to the application results conducted with RVR-I coefficients. In the denoising application conducted with CVR-I coefficients, the most successful results were obtained for the values between " 2.2 " and "2.5" of thresholding constant. Also, the PSNR values obtained within this range were the best results achieved in these denoising applications.

A number of original retinal images used in the processes, their noise-applied states for three different sigma values and their re-obtained states after denoising application for different support, degree values are shown in Figure 4.

Table 5. Results of PSNR for retinal images (Sigma=35, $a$ (Scale), c (Support), $d$ (Degree) and $k$ (Thresholding Constant)).

\begin{tabular}{|c|c|c|c|c|c|c|c|c|}
\cline { 2 - 8 } \multicolumn{1}{c|}{} & \multicolumn{4}{c|}{ Reel valued ripplet-I transform } & \multicolumn{3}{c|}{ Complex valued ripplet-I transform } \\
\cline { 2 - 9 } \multicolumn{1}{c|}{} & $c=1, d=2$ & $c=1, d=3$ & $c=1, d=4$ & $c=1, d=5$ & $c=1, d=2$ & $c=1, d=3$ & $c=1, d=4$ & $c=1, d=5$ \\
\hline$a=7$ & $32.75(k=3.1)$ & $32.48(k=3.0)$ & $32.27(k=3.0)$ & $32.17(k=3.1)$ & $33.05(k=2.4)$ & $32.78(k=2.4)$ & $32.56(k=2.3)$ & $32.42(k=2.4)$ \\
\hline$a=6$ & $32.97(k=3.1)$ & $32.83(k=3.1)$ & $32.68(k=3.1)$ & $32.67(k=3.1)$ & $33.24(k=2.4)$ & $33.11(k=2.4)$ & $32.95(k=2.4)$ & $32.95(k=2.4)$ \\
\hline$a=5$ & $33.04(k=3.1)$ & $33.00(k=3.1)$ & $33.02(k=3.1)$ & $33.02(k=3.1)$ & $33.30(k=2.4)$ & $33.25(k=2.5)$ & $33.27(k=2.5)$ & $33.27(k=2.5)$ \\
\hline$a=4$ & $32.16(k=3.3)$ & $32.17(k=3.3)$ & $32.17(k=3.3)$ & $32.17(k=3.3)$ & $32.37(k=2.5)$ & $32.39(k=2.5)$ & $32.39(k=2.5)$ & $32.39(k=2.5)$ \\
\hline
\end{tabular}




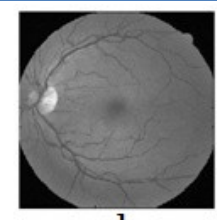

al
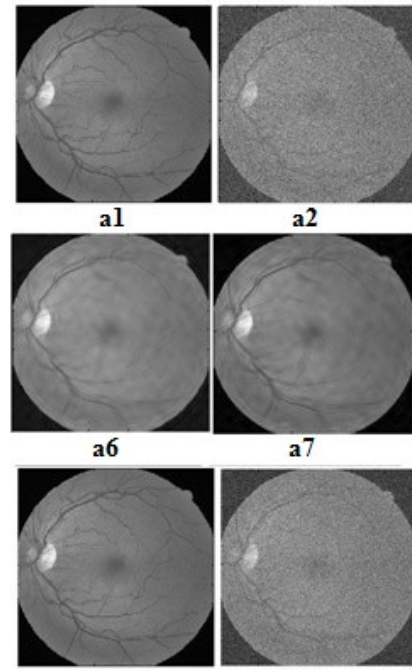

bl

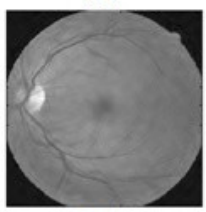

b6

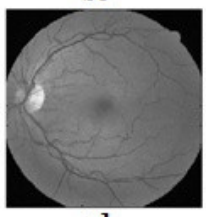

cl

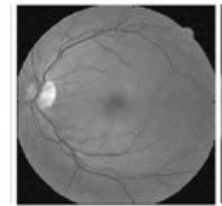

c6

a2

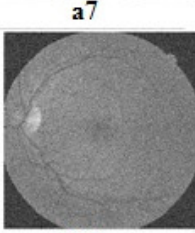

b2

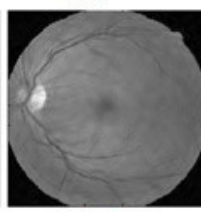

b7

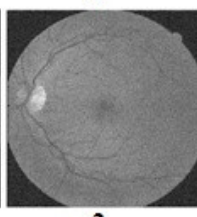

c2

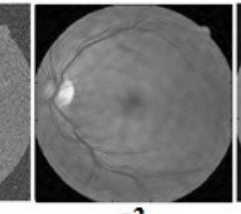

a3

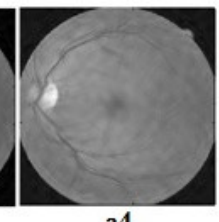

a4

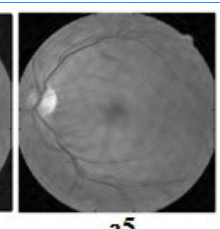

a5

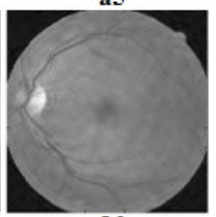

al0

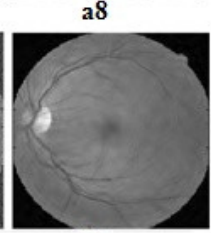

b3

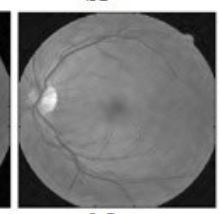

b8

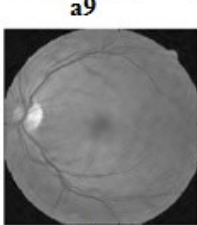

b4

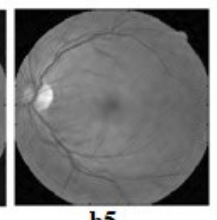

b5

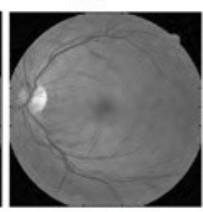

b9

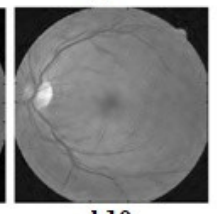

b10

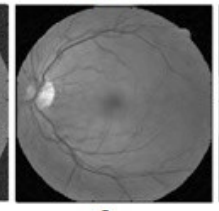

c3

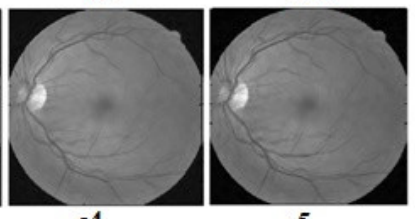

c5

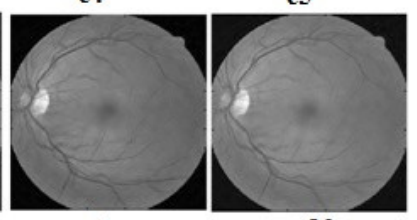

c9

c10

Figure 4. a1) Original retinal image a2) Noisy image (Sigma=35) a3) Denoising with RVR-I (c=1, $d=2)$ a4)

Denoising with CVR-I $(c=1, d=2)$ a5) Denoising with RVR-I $(c=1, d=3)$ a6) Denoising with CVR-I $(c=1, d=3)$ a7)

Denoising with RVR-I ( $c=1, d=4)$ a8) Denoising with CVR-I $(c=1, d=4)$ a9) Denoising with RVR-I $(c=1, d=5)$ a10) Denoising with CVR-I $(c=1, d=5)$ b1) Original retinal image b2) Noisy image (Sigma =25) b3) Denoising with RVR-I $(c=1, d=2)$ b4) Denoising with CVR-I $(c=1, d=2)$ b5) Denoising with RVR-I $(c=1, d=3)$ b6) Denoising with CVR-I $(c=1$, $d=3)$ b7) Denoising with RVR-I $(c=1, d=4)$ b8) Denoising with CVR-I $(c=1, d=4)$ b9) Denoising with RVR-I $(c=1, d=5)$ b10) Denoising with CVR-I $(c=1, d=5) c 1$ ) Original retinal image $c 2)$ Noisy image (Sigma=15) c3) Denoising with RVR-I $(c=1, d=2)$ c4) Denoising with CVR-I $(c=1, d=2)$ c5) Denoising with RVR-I $(c=1, d=3)$ c6) Denoising with CVR-I $(c=1, d=3)$ c7) Denoising with RVR-I $(c=1, d=4)$ c8) Denoising with CVR-I $(c=1, d=4)$ c9) Denoising with RVR-I $(c=1$, $d=5)$ c10) Denoising with CVR-I $(c=1, d=5)$ 
Table 6. Results of PSNR for retinal images (Sigma=25, $a$ (Scale), $c$ (Support), $d$ (Degree) and $k$ (Thresholding Constant)).

\begin{tabular}{|c|c|c|c|c|c|c|c|c|}
\cline { 2 - 8 } \multicolumn{1}{c|}{} & \multicolumn{4}{c|}{ Reel valued ripplet-I transform } & \multicolumn{4}{c|}{ Complex valued ripplet-I transform } \\
\cline { 2 - 9 } \multicolumn{1}{c|}{} & $c=1, d=2$ & $c=1, d=3$ & $c=1, d=4$ & $c=1, d=5$ & $c=1, d=2$ & $c=1, d=3$ & $c=1, d=4$ & $c=1, d=5$ \\
\hline$a=7$ & $34.08(k=3.0)$ & $33.84(k=3.0)$ & $33.65(k=2.9)$ & $33.49(k=2.9)$ & $34.31(k=2.4)$ & $34.08(k=2.3)$ & $33.87(k=2.3)$ & $33.67(k=2.3)$ \\
\hline$a=6$ & $34.29(k=3.0)$ & $34.13(k=3.0)$ & $33.99(k=3.0)$ & $33.98(k=3.0)$ & $34.48(k=2.4)$ & $34.35(k=2.4)$ & $34.21(k=2.4)$ & $34.20(k=2.4)$ \\
\hline$a=5$ & $34.39(k=3.0)$ & $34.35(k=3.0)$ & $34.35(k=3.0)$ & $34.35(k=3.0)$ & $34.58(k=2.4)$ & $34.54(k=2.4)$ & $34.54(k=2.4)$ & $34.54(k=2.4)$ \\
\hline$a=4$ & $33.89(k=3.1)$ & $33.92(k=3.1)$ & $33.92(k=3.1)$ & $33.92(k=3.1)$ & $34.04(k=2.5)$ & $34.08(k=2.4)$ & $34.08(k=2.4)$ & $34.08(k=2.4)$ \\
\hline
\end{tabular}

Table 7. Results of PSNR for retinal images (Sigma=15, a (Scale), c (Support), d (Degree) and k (Thresholding Constant)).

\begin{tabular}{|c|c|c|c|c|c|c|c|c|}
\cline { 2 - 8 } \multicolumn{1}{c|}{} & \multicolumn{4}{|c|}{ Reel valued ripplet-I transform } & \multicolumn{4}{c|}{ Complex valued ripplet-I transform } \\
\cline { 2 - 9 } \multicolumn{1}{c|}{} & $c=1, d=2$ & $c=1, d=3$ & $c=1, d=4$ & $c=1, d=5$ & $c=1, d=2$ & $c=1, d=3$ & $c=1, d=4$ & $c=1, d=5$ \\
\hline$a=7$ & $35.98(k=2.8)$ & $35.76(k=2.8)$ & $35.59(k=2.8)$ & $35.45(k=2.7)$ & $36.10(k=2.2)$ & $35.89(k=2.2)$ & $35.71(k=2.2)$ & $35.55(k=2.2)$ \\
\hline$a=6$ & $36.15(k=2.8)$ & $35.98(k=2.8)$ & $35.86(k=2.8)$ & $35.82(k=2.8)$ & $36.28(k=2.2)$ & $36.10(k=2.2)$ & $35.99(k=2.2)$ & $35.95(k=2.2)$ \\
\hline$a=5$ & $36.32(k=2.9)$ & $36.23(k=2.8)$ & $36.23(k=2.8)$ & $36.23(k=2.8)$ & $36.44(k=2.3)$ & $36.34(k=2.3)$ & $36.33(k=2.3)$ & $36.33(k=2.3)$ \\
\hline$a=4$ & $36.22(k=2.9)$ & $36.23(k=2.9)$ & $36.21(k=2.8)$ & $36.21(k=2.8)$ & $36.33(k=2.3)$ & $36.33(k=2.3)$ & $36.31(k=2.3)$ & $36.31(k=2.3)$ \\
\hline
\end{tabular}

\subsection{Mammographic Image Denoising}

In the wake of study, the best denoising PSNR results obtained with 332 mammographic images for three different sigma values $(15,25,25)$ were given in Table 8-10. Mammographic images are different then liver and retinal images in terms of the structure. The pixel values belonging to the breast tissue are very close to each other in mammographic images. This situation makes distinguishing the noises applied to images and the breast tissue structures from each other more difficult. Upon examining Table 8-10, the most successful results were obtained for several different scale values, unlike the other two groups where it is constant. But, again, it was gathered from the results obtained for mammographic images that the thresholding constant requires to be used differently in processes conducted with RVR-I and CVR-I coefficients. The most successful results were obtained for the values between " 3.0 " and "3.4" of thresholding constant in the denoising application conducted with RVR-I coefficients for mammographic images. In the denoising application conducted with CVR-I coefficients, the most successful results were obtained for the values between "2.3" and "2.7" of thresholding constant. Also, the PSNR values obtained within this range were the best results achieved in these denoising applications.

A number of original mammographic images used in the processes, their noise-applied states for three different sigma values and their re-obtained states after denoising application for different support, degree values are shown in Figure 5. 

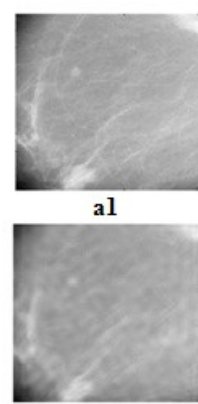

a6

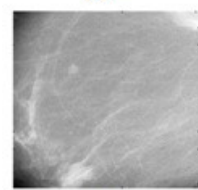

bl

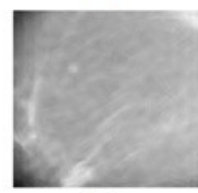

b6

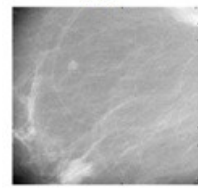

cl

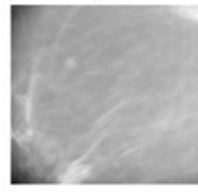

c6

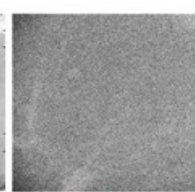

a2

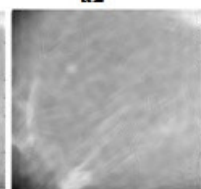

a7

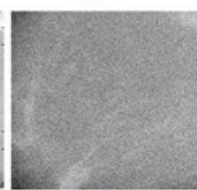

b2
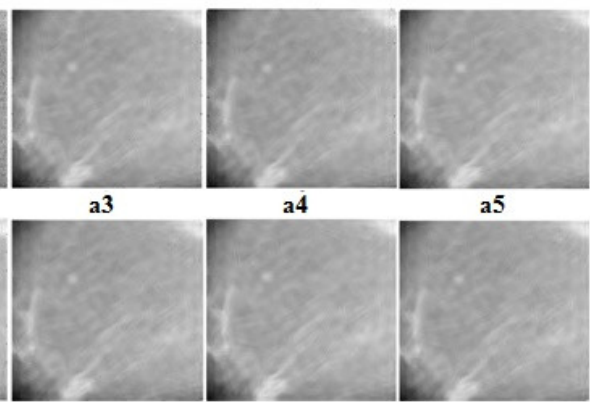

a8

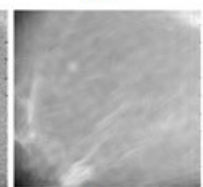

b3

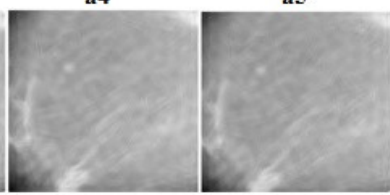

al0

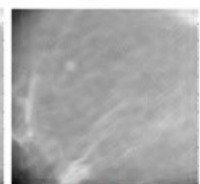

b4

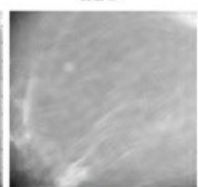

b5

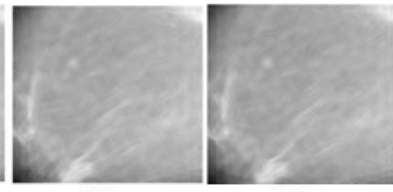

b8

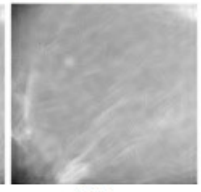

b9

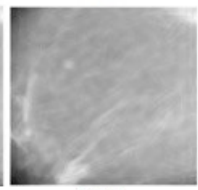

b10

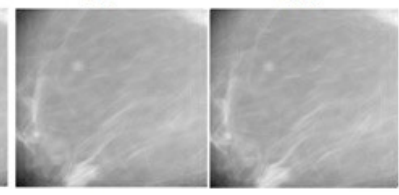

c5

c3

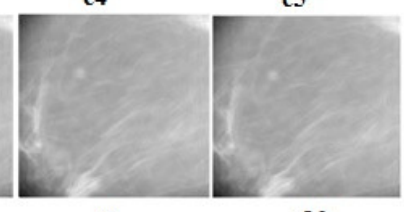

c9

Figure 5. a1) Original mammographic image a2) Noisy image (Sigma=35) a3) Denoising with $R V R-I(c=1, d=2)$ a4) Denoising with CVR-I (c=1, $d=2)$ a5) Denoising with RVR-I $(c=1, d=3)$ a6) Denoising with CVR-I $(c=1, d=3)$ a7) Denoising with RVR-I ( $c=1, d=4)$ a8) Denoising with CVR-I $(c=1, d=4)$ a9) Denoising with RVR-I $(c=1, d=5)$ a10) Denoising with CVR-I $(c=1, d=5)$ b1) Original mammographic image b2) Noisy image (Sigma=25) b3) Denoising with RVR-I (c=1, $d=2)$ b4) Denoising with CVR-I ( $c=1, d=2)$ b5) Denoising with RVR-I (c=1, d=3) b6) Denoising with CVR-I (c=1, $d=3)$ b7) Denoising with RVR-I (c=1, $d=4)$ b8) Denoising with CVR-I (c=1, d=4) b9) Denoising with RVR-I $(c=1, d=5)$ b10) Denoising with CVR-I $(c=1, d=5)$ c1) Original mammographic image c2) Noisy image (Sigma=15) c3) Denoising with RVR-I $(c=1, d=2)$ c4) Denoising with CVR-I $(c=1, d=2)$ c5) Denoising with RVR-I $(c=1, d=3)$ c6) Denoising with CVR-I $(c=1, d=3)$ c7) Denoising with RVR-I $(c=1, d=4)$ c8) Denoising with CVR-I $(c=1, d=4) c 9)$ Denoising with RVR-I (c=1, $d=5)$ c10) Denoising with CVR-I $(c=1, d=5)$ 
Journal of Biomedical Engineming and Medical Imaging, Volume 4, Issue 2, April, 2017

Table 8. Results of PSNR for mammographic images (Sigma=35, $a$ (Scale), $c$ (Support), $d$ (Degree) and $k$ (Thresholding Constant)).

\begin{tabular}{|c|c|c|c|c|c|c|c|c|}
\cline { 2 - 8 } \multicolumn{1}{c|}{} & \multicolumn{4}{c|}{ Reel valued ripplet-I transform } & \multicolumn{4}{c|}{ Complex valued ripplet-I transform } \\
\cline { 2 - 9 } \multicolumn{1}{c|}{} & $c=1, d=2$ & $c=1, d=3$ & $c=1, d=4$ & $c=1, d=5$ & $c=1, d=2$ & $c=1, d=3$ & $c=1, d=4$ & $c=1, d=5$ \\
\hline$a=7$ & $35.25(k=3.3)$ & $35.44(k=3.4)$ & $35.46(k=3.4)$ & $35.52(k=3.4)$ & $35.51(k=2.6)$ & $35.71(k=2.6)$ & $35.71(k=2.6)$ & $35.75(k=2.6)$ \\
\hline$a=6$ & $35.11(k=3.4)$ & $35.35(k=3.4)$ & $35.46(k=3.4)$ & $35.59(k=3.4)$ & $35.32(k=2.6)$ & $35.58(k=2.6)$ & $35.66(k=2.6)$ & $35.82(k=2.6)$ \\
\hline$a=5$ & $34.94(k=3.4)$ & $35.13(k=3.4)$ & $35.26(k=3.4)$ & $35.26(k=3.4)$ & $35.18(k=2.6)$ & $35.31(k=2.7)$ & $35.45(k=2.7)$ & $35.45(k=2.7)$ \\
\hline$a=4$ & $33.35(k=3.4)$ & $33.39(k=3.4)$ & $33.39(k=3.4)$ & $33.39(k=3.4)$ & $33.50(k=2.6)$ & $33.55(k=2.6)$ & $33.55(k=2.6)$ & $33.55(k=2.6)$ \\
\hline
\end{tabular}

Table 9. Results of PSNR for mammographic images (Sigma=25, $a$ (Scale), $c$ (Support), $d$ (Degree) and $k$ (Thresholding Constant)).

\begin{tabular}{|l|l|l|l|l|l|l|l|l|}
\cline { 2 - 8 } \multicolumn{4}{c|}{} & \multicolumn{4}{l}{ Reel valued ripplet-I transform } & \multicolumn{3}{l|}{ Complex valued ripplet-I transform } \\
\cline { 2 - 9 } \multicolumn{1}{l|}{} & $c=1, d=2$ & $c=1, d=3$ & $c=1, d=4$ & $c=1, d=5$ & $c=1, d=2$ & $c=1, d=3$ & $c=1, d=4$ & $c=1, d=5$ \\
\hline$a=7$ & $36.51(k=3.2)$ & $36.60(k=3.2)$ & $36.61(k=3.2)$ & $36.65(k=3.2)$ & $36.72(k=2.4)$ & $36.79(k=2.5)$ & $36.80(k=2.5)$ & $36.80(k=2.5)$ \\
\hline$a=6$ & $36.35(k=3.3)$ & $36.57(k=3.2)$ & $36.64(k=3.2)$ & $36.71(k=3.3)$ & $36.55(k=2.4)$ & $36.76(k=2.4)$ & $36.82(k=2.5)$ & $36.89(k=2.5)$ \\
\hline$a=5$ & $36.34(k=3.2)$ & $36.46(k=3.3)$ & $36.61(k=3.3)$ & $36.61(k=3.3)$ & $36.59(k=2.5)$ & $36.64(k=2.6)$ & $36.81(k=2.6)$ & $36.81(k=2.6)$ \\
\hline$a=4$ & $35.34(k=3.4)$ & $35.47(k=3.4)$ & $35.47(k=3.4)$ & $35.47(k=3.4)$ & $35.47(k=2.6)$ & $35.63(k=2.6)$ & $35.63(k=2.6)$ & $35.63(k=2.6)$ \\
\hline
\end{tabular}

Table 10. Results of PSNR for mammographic images (Sigma=15, $a$ (Scale), $c$ (Support), $d$ (Degree) and $k$ (Thresholding Constant)).

\begin{tabular}{|c|c|c|c|c|c|c|c|c|}
\cline { 2 - 8 } \multicolumn{1}{c|}{} & \multicolumn{4}{c|}{ Reel valued ripplet-I transform } & \multicolumn{4}{c|}{ Complex valued ripplet-I transform } \\
\cline { 2 - 9 } \multicolumn{1}{c|}{} & $c=1, d=2$ & $c=1, d=3$ & $c=1, d=4$ & $c=1, d=5$ & $c=1, d=2$ & $c=1, d=3$ & $c=1, d=4$ & $c=1, d=5$ \\
\hline$a=7$ & $38.25(k=3.0)$ & $38.30(k=3.0)$ & $38.29(k=3.0)$ & $38.31(k=2.9)$ & $38.41(k=2.4)$ & $38.44(k=2.3)$ & $38.43(k=2.3)$ & $38.44(k=2.3)$ \\
\hline$a=6$ & $38.17(k=2.9)$ & $38.29(k=3.0)$ & $38.32(k=3.0)$ & $38.37(k=3.0)$ & $38.35(k=2.3)$ & $38.43(k=2.4)$ & $38.44(k=2.4)$ & $38.49(k=2.4)$ \\
\hline$a=5$ & $38.17(k=3.1)$ & $38.22(k=3.1)$ & $38.32(k=3.1)$ & $38.32(k=3.1)$ & $38.36(k=2.3)$ & $38.38(k=2.4)$ & $38.46(k=2.4)$ & $38.46(k=2.4)$ \\
\hline$a=4$ & $37.87(k=3.1)$ & $38.02(k=3.2)$ & $38.02(k=3.2)$ & $38.02(k=3.2)$ & $38.00(k=2.5)$ & $38.17(k=2.5)$ & $38.17(k=2.5)$ & $38.17(k=2.5)$ \\
\hline
\end{tabular}

\section{Conclusions}

In this study, the medical image denoising application was realized by using RVR-I and CVR-I coefficients. Formerly it was known that using complex coefficients from transforms such as wavelet, ridgelet gave better results in denoising applications compared to real coefficients. Thanks to this study, it was verified that it is also viable for the ripplet-I transform. Table 2-10 shows that the PSNR results obtained by using CVR-I coefficients for all the scale, support, degree values gave better results that the ones obtained by using RVR-I coefficients. In addition, detailed results of MSSIM and FSIM seem to support this case. Table 11 and 12 shows the best MSSIM and FSIM results obtained for different scale, support, degree and thresholding constant values in the wake of study by using liver, retinal and mammographic images. 
Hüseyin Yașar, Murat Ceylan; Real and Complex Valued Ripplet-I Transform for Medical Image Denoising and Analysis of Thresholding Constants and Scales Effects . Journal of Biomedical Engineering and Medical Imaging, Volume 4, No 2, April (2017), pp 10-30

Table 11. Results of the highest MSSIM (c (Support), $d$ (Degree)).

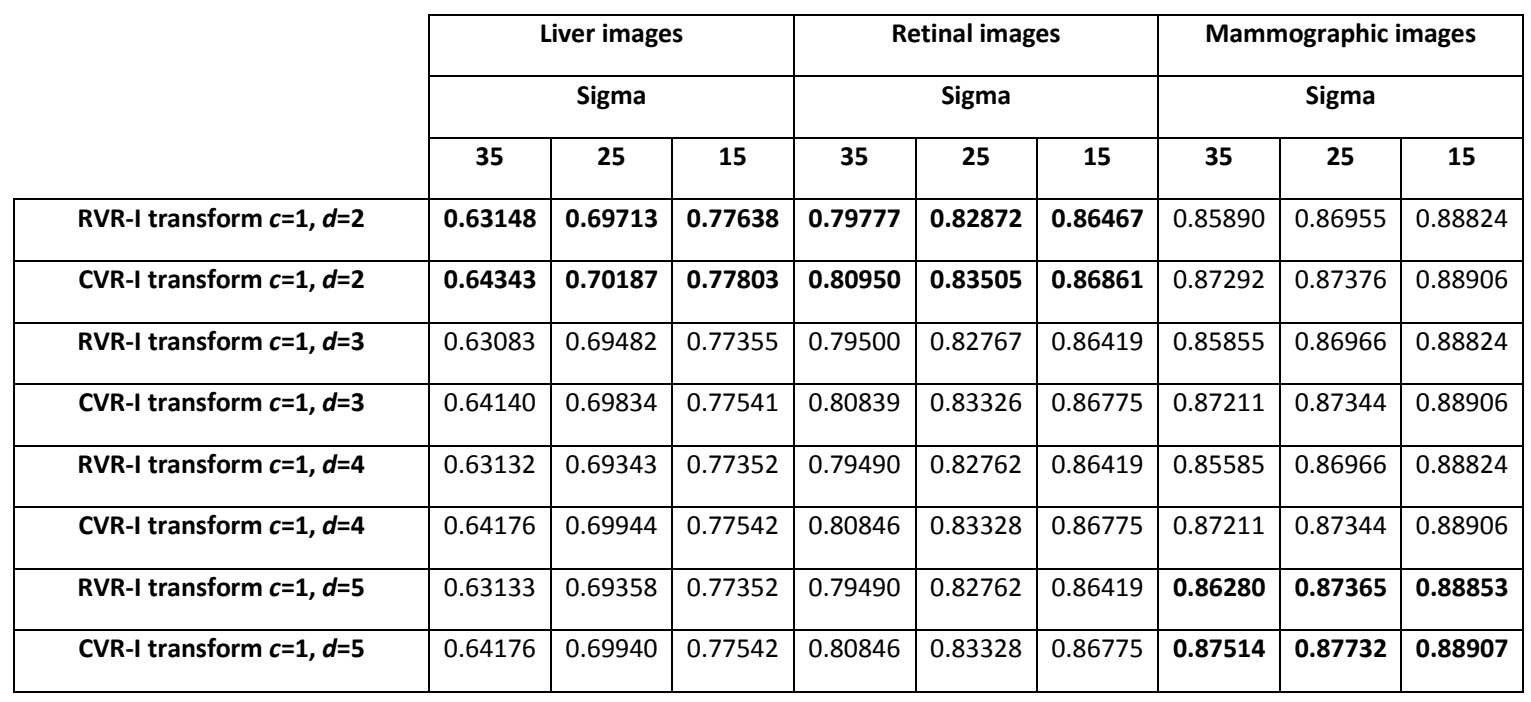

Table 12. Results of the highest FSIM (c (Support), d (Degree))

\begin{tabular}{|c|c|c|c|c|c|c|c|c|c|}
\hline & \multicolumn{3}{|c|}{ Liver images } & \multicolumn{3}{|c|}{ Retinal images } & \multicolumn{3}{|c|}{ Mammographic images } \\
\hline & \multicolumn{3}{|c|}{ Sigma } & \multicolumn{3}{|c|}{ Sigma } & \multicolumn{3}{|c|}{ Sigma } \\
\hline & 35 & 25 & 15 & 35 & 25 & 15 & 35 & 25 & 15 \\
\hline RVR-I transform $c=1, d=2$ & 0.87287 & 0.90176 & 0.93815 & 0.89022 & 0.91513 & 0.94659 & 0.91049 & 0.92354 & 0.94851 \\
\hline CVR-I transform $c=1, d=2$ & 0.88831 & 0.91388 & 0.94576 & 0.89398 & 0.91743 & 0.94712 & 0.91079 & 0.92558 & 0.94886 \\
\hline RVR-I transform $c=1, d=3$ & 0.87229 & 0.90121 & 0.93723 & 0.88995 & 0.91460 & 0.94650 & 0.91104 & 0.92410 & 0.94867 \\
\hline CVR-I transform $c=1, d=3$ & 0.88792 & 0.91358 & 0.94510 & 0.89372 & 0.91711 & 0.94703 & 0.91125 & 0.92626 & 0.94901 \\
\hline RVR-I transform $c=1, d=4$ & 0.87240 & 0.90126 & 0.93727 & 0.89000 & 0.91463 & 0.94650 & 0.91262 & 0.92498 & 0.94867 \\
\hline CVR-I transform $c=1, d=4$ & 0.88797 & 0.91358 & 0.94509 & 0.89379 & 0.91711 & 0.94703 & 0.91279 & 0.92702 & 0.94901 \\
\hline RVR-I transform $c=1, d=5$ & 0.87240 & 0.90126 & 0.93727 & 0.89000 & 0.91463 & 0.94650 & 0.91349 & 0.92509 & 0.94867 \\
\hline CVR-I transform $c=1, d=5$ & 0.88797 & 0.91358 & 0.94509 & 0.89379 & 0.91711 & 0.94703 & 0.91387 & 0.92723 & 0.94902 \\
\hline
\end{tabular}

This study helped examine the effects that the change of the scale value has on the medical image denoising results. Upon examining Table 2-10, the image denoising algorithm used in study gave the best results so long as the scale value was " 5 " for all the support and degree values in liver and retinal images. However in mammographic images, the scale value from which the best results were obtained varied.

This study also examined the effects that the change of the thresholding constant has on the image denoising results. This phase suggested that the values to be used generally when processing with complex coefficients need to be chosen different from when processing with real coefficients of thresholding constant. Upon examining Table 2-10, the best results in the denoising applications conducted with real coefficients were obtained for the thresholding constant values between "2.7" and "3.4" in the wake of study. In the previously conducted real valued applications, the thresholding constant was used in general as " 3 " and this situation corresponds to the obtained results. Upon 
examining Table 2-10, it was reached as a result of the study that in the denoising applications conducted with complex coefficients, the thresholding constant needs to be of a lower value, namely picked between "2.2" and "2.7".

According to the study results, it is important to examine the effect that the scale change has on the change of the thresholding constant value where the best denoising result is obtained. The thresholding constant that which produces the most successful result tends to increase as the scale value in the denoising application conducted with RVR-I and CVR-I coefficients for liver, retinal and mammographic images decreases. Figure 6 shows the change in the average of the thresholding constant values, where the best denoising results are obtained; this change is based on the scale value.
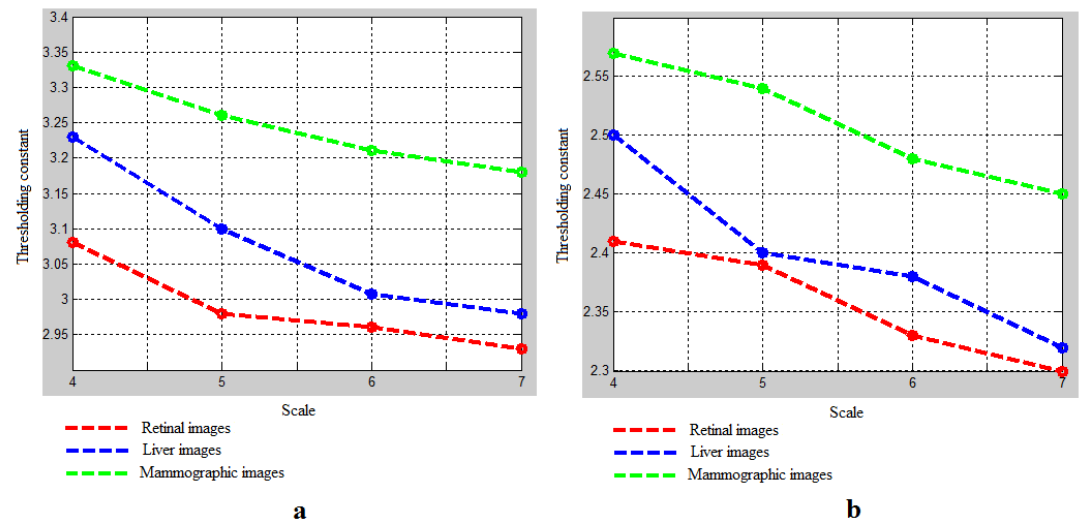

Figure 6. Change in the average of the thresholding constant values, where the best denoising results are obtained, based on the scale value a) Denoising with RVR-I coefficients b) Denoising with CVR-I coefficients

Table 13, shows the best PSNR results obtained for different scale, support, degree and thresholding constant values in the wake of study by using liver, retinal and mammographic images. Upon examining Table 13, it can be seen that the best results for liver, retinal images were obtained for $c=1, d=2$ values; whereas for mammographic images, the best results were obtained for $c=1, d=5$ values.

Upon examining Table 13, it can be seen that the change between the best results obtained for $c=1, d=3$; $c=1, d=4 ; c=1, d=5$ values with liver and retinal images is very small. The best results obtained for these images having been achieved, as the scale value was " 5 ", had an effect on the occurrence of this situation. Upon examining the decompositions given in Table I, it was observed that as the scale value increased, decompositions diversified. The best results obtained for these images having been achieved, as the scale value was " 5 " instead of a bigger value, caused the decompositions to diversify less and the best results that were obtained correspondingly to be close to each other. Also for mammographic images, the fact that their best results had been obtained for different scale values verifies this change as significant. Therefore, the best results for mammographic images were obtained for $c=1, d=5$ values. This being the case suggests that the scale, support and degree value preference should be determined based on the image to be denoised.

Based on the developments made in the field of multi-resolution analysis, there were also developments in the field of medical image denoising. The ripplet-I transform was realized by generalizing the curvelet transform. Again, in later studies, the ripplet-II transform was realized by generalizing the ridgelet 
Hüseyin Yaşar, Murat Ceylan; Real and Complex Valued Ripplet-I Transform for Medical Image Denoising and Analysis of Thresholding Constants and Scales Effects . Journal of Biomedical Engineering and Medical Imaging, Volume 4, No 2, April (2017), pp 10-30

transform. With this study, by using the complex valued ripplet-I coefficients for the first time, the medical image denoising application was realized. Later on, conducting the medical image denoising application by forming complex coefficients in the ripplet-II transform is viewed as a big contribution to the literature.

Table 13. Results of the highest PSNR (c (Support), $d$ (Degree))

\begin{tabular}{|c|c|c|c|c|c|c|c|c|c|}
\hline & \multicolumn{3}{|c|}{ Liver images } & \multicolumn{3}{|c|}{ Retinal images } & \multicolumn{3}{|c|}{ Mammographic images } \\
\hline & \multicolumn{3}{|c|}{ Sigma } & \multicolumn{3}{|c|}{ Sigma } & \multicolumn{3}{|c|}{ Sigma } \\
\hline & 35 & 25 & 15 & 35 & 25 & 15 & 35 & 25 & 15 \\
\hline RVR-I transform $c=1, d=2$ & 34.460 & 36.080 & 38.656 & 33.042 & 34.387 & 36.322 & 35.247 & 36.508 & 38.254 \\
\hline CVR-I transform $c=1, d=2$ & 34.753 & 36.363 & 38.934 & 33.300 & 34.579 & 36.443 & 35.510 & 36.719 & 38.412 \\
\hline RVR-I transform $c=1, d=3$ & 34.434 & 36.045 & 38.605 & 33.005 & 34.345 & 36.234 & 35.444 & 36.603 & 38.303 \\
\hline CVR-I transform $c=1, d=3$ & 34.709 & 36.341 & 38.862 & 33.246 & 34.540 & 36.340 & 35.711 & 36.794 & 38.445 \\
\hline RVR-I transform $c=1, d=4$ & 34.441 & 36.051 & 38.611 & 33.022 & 34.348 & 36.234 & 35.463 & 36.640 & 38.320 \\
\hline CVR-I transform $c=1, d=4$ & 34.710 & 36.341 & 38.863 & 33.270 & 34.543 & 36.329 & 35.713 & 36.815 & 38.460 \\
\hline RVR-I transform $c=1, d=5$ & 34.441 & 36.051 & 38.611 & 33.022 & 34.348 & 36.234 & 35.588 & 36.714 & 38.373 \\
\hline CVR-I transform $c=1, d=5$ & 34.710 & 36.341 & 38.863 & 33.270 & 34.543 & 36.329 & 35.820 & 36.892 & 38.488 \\
\hline
\end{tabular}

\section{ACKNOWLEDGMENT}

This study was supported by The Scientific and Technical Research Council of Turkey (TUBITAK, Project No: 113E184). The liver images used in this study were taken from the database of this project.

\section{REFERENCES}

[1]. Reider, P. and Nossek, J. A. Implementation of orthogonal wavelet transforms and their applications. in Application-Specific Systems, Architectures and Processors, 1997. Proceedings. IEEE International Conference on.

[2]. Pu-chun, C., et al., Application of Debauchies wavelet to the de-noising of radio maps. Chinese Astronomy and Astrophysics, 2001. 25(1): p. 132-137.

[3]. Zhai, J. and Zhang, S. Image denoising via wavelet threshold: single wavelet and multiple wavelets transform. in Machine Learning and Cybernetics, 2005. Proceedings of 2005 International Conference on.

[4]. Li, X. and Zhu, S. Image denoising based on wavelet domain spatial context modeling. in Intelligent Control and Automation, 2006. WCICA 2006. The Sixth World Congress on.

[5]. Junli, L., et al. A novel method for the determination of the wavelet denoising threshold. in Bioinformatics and Biomedical Engineering, 2007. ICBBE 2007. The 1st International Conference on.

[6]. Hongqiao, L. and Shengqian, W. A new image denoising method using wavelet transform. in Information Technology and Applications, 2009. IFITA'09. International Forum on. 
[7]. Wang, X. Y., et al., A new wavelet-based image denoising using undecimated discrete wavelet transform and least squares support vector machine. Expert Systems with Applications, 2010. 37(10): p. 7040-7049.

[8]. Chen, G., et al., Wavelet-based image denoising using three scales of dependency. IET Image Process, 2012. 6(6): p. 756-760.

[9]. Chen, G., et al. Wavelet-based denoising: a brief review. in Intelligent Control and Information Processing (ICICIP), 2013 Fourth International Conference on.

[10]. Candes, E. J. and Donoho, D. L., Ridgelets: the key to high-dimensional intermittency. Philosophical Transactions Royal Society, 1999. 357: p. 2495-2509.

[11]. Candes, E. J. and Donoho, D. L., Curvelets-A surprisingly effective nonadaptie representation for objects with edges. Chap. Curves and Surface Fitting, A. Cohen, C. Rabut, L. Schumaker, Editors 1999. p. 105-120.

[12]. Starck, J. L., et al., The curvelet transform for image denoising. IEEE Transactions on Image Processing, 2002. 11(6): p. 670-684.

[13]. Sivakumar, R., Denoising of computer tomography images using curvelet transform. ARPN Journal of Engineering and Applied Sciences, 2007. 2(1): p. 21-26.

[14]. Ma, L., et al., Pixel fusion based curvelets and wavelets denoise algorithm. Engineering Letters, 2007. 14(2): p. 130-134.

[15]. Ali, F. E., et al., Curvelet fusion of MR and CT images. Progress In Electromagnetics Research C, 2008. 3: p. $215-224$.

[16]. Reddy, G. J., et al., Fingerprint image denoising using curvelet transform. ARPN Journal of Engineering and Applied Sciences, 2008. 3(3): p. 31-35.

[17]. Patil, A. A. and Singhai, J., Image denoising using curvelet transform: an approach for edge preservation. Journal of Scientific \& Industrial Research, 2010. 69: p. 34-38.

[18]. Binh, N. T. and Khare, A., Multilevel threshold based image denoising in curvelet domain. Journal of Computer Science and Technology, 2010. 25(3): p. 632-640.

[19]. Shukla, M. and Changlani, S., A comparative study of wavelet and curvelet transform for image denoising. IOSR Journal of Electronics and Communication Engineering, 2013. 7(4): p. 63-68.

[20]. Candes, E. J. and Donoho, D. L., New tight frames of curvelets and optimal representations of objects with piecewise-C2 singularities. Communications on Pure and Applied Mathematics, 1999. 57(2): p. 219-266.

[21]. Candes, E. J. and Guo, F., New multiscale transforms, minimum total variation synthesis: application to edgepreserving image reconstruction. Sig. Process., special issue on Image and Video Coding Beyond Standards, 2002. 82(11): p. 1519-1543.

[22]. Lawton, W., Applications of complex valued wavelet transforms to subband decomposition. IEEE Trans. Signal Processing, 1993. 41(12): p. 3566-3568.

[23]. Lina, J. M., Complex Daubechies wavelets: filter design and applications. Inverse Problems, Tomography, and Image Processing, 1998. p. 95-112. 
Hüseyin Yaşar, Murat Ceylan; Real and Complex Valued Ripplet-I Transform for Medical Image Denoising and Analysis of Thresholding Constants and Scales Effects. Journal of Biomedical Engineering and Medical Imaging, Volume 4, No 2, April (2017), pp 10-30

[24]. Selesnick, I. W., et al., The dual-tree complex wavelet transform. IEEE Signal Processing Magazine, 2005. 22(6): p. 123-151.

[25]. Aryaz, B. and Runyi, Y. A dual-tree complex wavelet with application in image denoising. in Signal Processing and Communications, 2007. ICSPC 2007. IEEE International Conference on.

[26]. Jingyu, Y., et al. 2-D anisotropic dual-tree complex wavelet packets and its application to image denoising. in Image Processing, 2008. ICIP 2008. 15th IEEE International Conference on.

[27]. Song, Z. and Yuanpeng, L. A novel image denosing scheme via combining dual-tree complex wavelet transform and bandelets. in Intelligent Information Technology Application, 2009. IITA 2009. Third International Symposium on.

[28]. Candes, E. J., Fast discrete curvelet transforms. Multiscale Modeling \& Simulation, 2006. 5(3): p. 861-899.

[29]. Neelamani, R., et al., Complex curvelet-based adaptive subtraction of several noise templates. in SEG Technical Program Expanded Abstracts 2008. Society of Exploration Geophysicists.

[30]. Neelamani, R., et al., Adaptive subtraction using complex curvelet transform. in 70th EAGE Conference and Exhibition incorporating SPE EUROPEC 2008.

[31]. Neelamani, R., et al., Adaptive subtraction using complex-valued curvelet transform. Geophysics, 2010. 75(4): $\mathrm{p}$. V51-V60.

[32]. Yan, H., et al. Image restoration using Gaussian scale mixtures in complex curvelet transform domain. in Measuring Technology and Mechatronics Automation (ICMTMA), 2010 International Conference on.

[33]. Xu, J., et al., Ripplet: A new transform for image processing. Journal of Visual Communication and Image Representation, 2010. 21(7): p. 627-639.

[34]. Gupta, D., et al., Ripplet domain non-linear filtering for speckle reduction in ultrasound medical images. Biomedical Signal Processing and Control, 2014. 10: p. 79-91.

[35]. Yaşar, H. and Ceylan, M. New approaches based on real and complex forms of ripplet-I transform for image analysis, in Signal Processing and Communication Application Conference, 2016.

[36]. Ceylan, M. and Yaşar, H., A novel approach for automatic blood vessel extraction in retinal images: complex ripplet-I transform and complex valued articial neural network. Turkish Journal of Electrical Engineering and Computer Sciences, 2016. 24(4): p. 3212--3227.

[37]. Yaşar, H., Analysis of medical images with multi-resolution methods, MA thesis, The Graduate School of Natural and Applied of Selcuk University, Advisor: M. Ceylan, 2015.

[38]. www.ece.rice.edu/ wakin/images. (accessed 18 February 2017).

[39]. www.curvelet.org. (accessed 18 February 2017).

[40]. Bao, O. Z., et al., Local adaptive shrinkage threshold denoising using curvelet coefficients. Electronics Letters, 2008. 44(4): p. 277-278. 
Journal of Biomedical Engineming and Medical Imaging, Volume 4, Issue 2, April, 2017

[41]. Staal, J., et al., Ridge based vessel segmentation in color images of the retina. IEEE Transactions on Medical Imaging, 2004. 23(4): p. 501-509.

[42]. Suckling, J., et al., The mammographic image analysis society digital mammogram database, in Exerpta Medica. International Congress Series, 1994.

[43]. Wang, Z. et al., Image quality assessment: from error measurement to structural similarity. IEEE Trans. Image Processing, 2004. 13(4): p. 600-612.

[44]. Thakur, N. and Devi, S.; A new method for color image quality assessment. International Journal of Computer Applications, 2011. 15(2):p. 10-17.

[45]. Zhang, L. et al., FSIM: A feature similarity index for image quality assessment. IEEE Trans. Image Processing, 2011. 20(8): p. 2378-2386. 